\title{
三维双重孔隙-裂隙介质热-水-应力-迁移堣合模型 及其有限元分析
}

\author{
张玉军 ${ }^{(1 *}$, 张维庆 ${ }^{(2)}$ \\ (1) 中国科学院武汉岩土力学研究所, 岩土力学与工程国家重点实验室, 武汉 430071; \\ (2) 西南交通大学土木工程学院, 成都 610031 \\ *E-mail: yjzhang@whrsm.ac.cn
}

收稿日期: 2009-10-29; 接受日期: 2010-04-29

国家重点基础研究发展计划(“973”计划)(批准号: 2010CB732101)和岩土力学与工程国家重点实验室前沿探索性项目(批准号: SKLQ008)资助

\begin{abstract}
摘要建立了一种饱和-非饱和的双重孔隙-裂隙介质热-水-应力-迁移耦合三维模型, 其特点是应力场和温度场是单一的, 但具有不同的孔隙渗流场、裂隙渗流场和孔隙浓 度场、裂隙浓度场，以及可考虑裂隙的组数、间距、方向、连通率和刚度对本构关系 的影响, 并研制出相应的三维有限元程序. 通过与已有算例的对比, 验证了该模型和 程序的可靠性. 针对一个假定的高放废物地质处置库, 就岩体和缓冲层均为非饱和介
\end{abstract}

关键词

双重孔隙-裂隙介质 热-水-应力-迁移耦合 三维模型

有限元分析

\section{1 引言}

在自然界中，类似于花岗岩和凝灰岩这样遍有 节理、同时具有岩石孔隙和岩体裂隙的介质可称为双 重孔隙-裂隙介质. 中国、日本、瑞典、瑞士和加拿大 等国家拟在花岗岩中进行高放射性核废物的地质处 置, 而美国已在内华达州的凝灰岩中完成了坑道规 模试验(DST), 并将在尤卡山(Yucca Mountain)地区 建成世界上第一个高放废物地质处置库 ${ }^{[1,2]}$. 近年来, 就双重孔隙-裂隙介质中存在的温度场、应力场、渗 流场的相互作用问题, 若干学者已开展了热-水-力耦
合课题的探讨 ${ }^{[3 \sim 6]}$. 笔者也曾就国内外类似研究工作 中的缺点作了改进, 建立了一种双重孔隙-裂隙介质 热-水-应力耦合模型 ${ }^{[7]}$, 其特点是较严格地推导了应 力平衡、水连续性及能量守恒三大控制方程, 全耦合 求解, 用于饱和-非饱和的遍有节理的岩体, 可考虑 裂隙的组数、间距、方向、连通率、开度、刚度的影 响,并研制出相应的二维有限元程序. 但该模型也不 够完善，即没有考虑核素迁移的耦合分析.

梁冰等人 ${ }^{[8]}$ 充分考虑了地下水变密度对核素浓 度分布的影响, 建立了孔隙-裂隙岩体中核素随地下 水迁移的耦合数值模型; Sonnenthal 等人 ${ }^{[9]}$ 使用 
TOUGHREACT 程序模拟了尤卡山坑道规模试验中 双重介质内部的热-水-化学过程. 不过这些研究还局 限于水-迁移耦合、或热-水-迁移(化学)耦合. 武文华、 刘泽佳等人 ${ }^{[10,11]}$ 曾提出和发展了多孔介质中的化学热-水力-力学耦合本构模型; 笔者也建立过一个饱和 -非饱和多孔介质中热-水-应力-迁移耦合模型 ${ }^{[12]}$. 但 该类模型只适用于单重孔隙介质和平面应变问题.

为此, 针对上述研究中的不足之处, 笔者在自己 以及前人已有研究成果的基础上, 首先在理论上发 展了双重孔隙-裂隙介质中热-水-应力-迁移耦合的数 学模型, 其次研制出相应的三维弹性有限元程序, 然 后从方法论研究的角度, 以一个假定的高放废物地 质处置库为算例, 在一定的初始温度、孔隙水压力、 岩体应力和核素释放强度条件下, 就岩体是非饱和 双重孔隙-裂隙介质的情况, 考察了处置库近场的温 度、孔隙水压力、水流速、核素浓度和正应力的分布 与变化, 得出了若干的认识.

\section{2 双重孔隙-裂隙介质热-水-应力-迁移塊 合方程}

对于图 1 所示的双重孔隙-裂隙介质, 认为其中 存在着孔隙水压力和裂隙水压力、孔隙浓度和裂隙浓 度, 但应力场和温度场是单一的, 从而建立了一种热水-应力-迁移耦合三维模型. 略去繁琐的推导过程, 给出控制方程如下.

\section{1 应力平衡方程}

借鉴了 Leiws 提出的在非饱和条件下有效应力 的计算方法 ${ }^{[13]}$, 并假定孔隙岩体中发育有 $n$ 组裂隙 (见图 2), 在整体坐标系中有

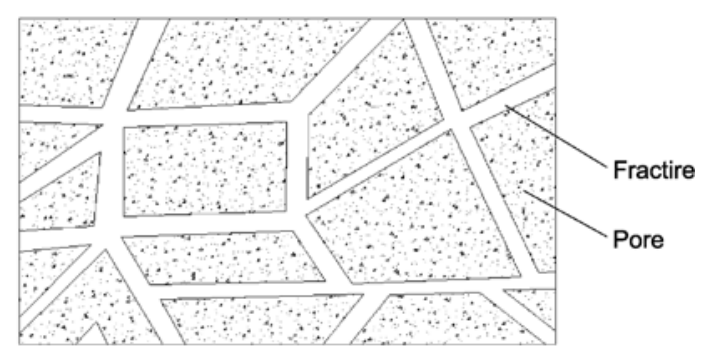

图 1 孔隙-裂隙介质

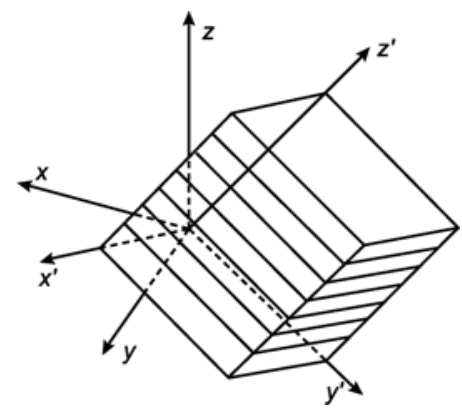

图 2 局部与整体坐标系

$$
\begin{gathered}
\frac{\mathrm{d} \boldsymbol{\sigma}}{\mathrm{d} t}=\boldsymbol{D}\left[\frac{\mathrm{d} \boldsymbol{\varepsilon}}{\mathrm{d} t}-\boldsymbol{m}\left(\boldsymbol{C}_{1}-\frac{1}{3 K_{s}}\right)\left(s_{w 1}+D_{s 1} p_{w 1}\right) \frac{\mathrm{d} p_{w 1}}{\mathrm{~d} t}\right. \\
\left.-\boldsymbol{m}\left(\boldsymbol{C}_{2}-\frac{1}{3 K_{s}}\right)\left(s_{w 2}+D_{s 2} p_{w 2}\right) \frac{\mathrm{d} p_{w 2}}{\mathrm{~d} t}-\boldsymbol{m} \frac{\beta_{S}}{3} \frac{\mathrm{d} T}{\mathrm{~d} t}\right],
\end{gathered}
$$

这里, $\sigma, \varepsilon$ 分别是总应力和总应变; $\boldsymbol{D}=\left(\boldsymbol{C}_{1}+\boldsymbol{C}_{2}\right)^{-1}$ 为弹

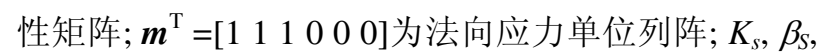
$T$ 依次是岩质固体的体积模量、综合热膨胀系数和温 度; $s_{w 1}, p_{w 1}, D_{s 1}, \boldsymbol{C}_{1}$ 和 $s_{w 2}, p_{w 2}, D_{s 2}, \boldsymbol{C}_{2}$ 分别是孔隙岩体 及裂隙介质中的饱和度、水压力、湿气容量和柔度矩 阵; $t$ 为时间. 不考虑负的孔隙水压力和裂隙水压力 对应力平衡的影响 ${ }^{[14]}$.

而且

$$
D_{s 1}=\frac{\partial s_{w 1}}{\partial p_{w 1}}, \quad D_{s 2}=\frac{\partial s_{w 2}}{\partial p_{w 2}} .
$$

对于三维问题, 有

$$
\begin{gathered}
\boldsymbol{C}_{1}=\frac{1}{E}\left[\begin{array}{cccccc}
1 & -\mu & -\mu & 0 & 0 & 0 \\
-\mu & 1 & -\mu & 0 & 0 & 0 \\
-\mu & -\mu & 1 & 0 & 0 & 0 \\
0 & 0 & 0 & 2(1+\mu) & 0 & 0 \\
0 & 0 & 0 & 0 & 2(1+\mu) & 0 \\
0 & 0 & 0 & 0 & 0 & 2(1+\mu)
\end{array}\right], \\
\boldsymbol{C}_{2 i}^{\prime}=\frac{A_{i}}{S_{i}}\left[\begin{array}{cccccc}
1 / K_{n i} & 0 & 0 & 0 & 0 & 0 \\
0 & 0 & 0 & 0 & 0 & 0 \\
0 & 0 & 0 & 0 & 0 & 0 \\
0 & 0 & 0 & 1 / K_{s x^{\prime} i} & 0 & 0 \\
0 & 0 & 0 & 0 & 1 / K_{s y^{\prime} i} & 0 \\
0 & 0 & 0 & 0 & 0 & 0
\end{array}\right],
\end{gathered}
$$




$$
\boldsymbol{L}_{i}=\left[\begin{array}{cccccc}
l_{1}^{2} & l_{2}^{2} & l_{3}^{2} & 2 l_{1} l_{2} & 2 l_{2} l_{3} & 2 l_{3} l_{1} \\
m_{1}^{2} & m_{2}^{2} & m_{3}^{2} & 2 m_{1} m_{2} & 2 m_{2} m_{3} & 2 m_{3} m_{1} \\
n_{1}^{2} & n_{2}^{2} & n_{3}^{2} & 2 n_{1} n_{2} & 2 n_{2} n_{3} & 2 n_{3} n_{1} \\
l_{1} m_{1} & l_{2} m_{2} & l_{3} m_{3} & l_{1} m_{2}+l_{2} m_{1} & l_{2} m_{3}+l_{3} m_{2} & l_{3} m_{1}+l_{1} m_{3} \\
m_{1} n_{1} & m_{2} n_{2} & m_{3} n_{3} & m_{1} n_{2}+m_{2} n_{1} & m_{2} n_{3}+m_{3} n_{2} & m_{3} n_{1}+m_{1} n_{3} \\
n_{1} l_{1} & n_{2} l_{2} & n_{3} l_{3} & n_{1} l_{2}+n_{2} l_{1} & n_{2} l_{3}+n_{3} l_{2} & n_{3} l_{1}+n_{1} l_{3}
\end{array}\right] .
$$

上述式子中, $E, \mu$ 分别为孔隙岩体的弹性模量和 泊桑系数; $\boldsymbol{C}_{2 i}^{\prime}$ 为局部坐标系中的裂隙柔度矩阵; $\boldsymbol{L}_{i}$ 为 坐标变换矩阵; $K_{n i}, K_{s x^{\prime} i}, K_{s y^{\prime}}, A_{i}, S_{i}$ 依次为裂隙的法向 刚度、沿 $x^{\prime}$ 方向的剪切刚度、沿 $y^{\prime}$ 方向的剪切刚度、 连通率和间距; $\left\{l_{i}, m_{i}, n_{i}, i=1,2,3\right\}$ 为局部坐标轴与 整体坐标轴之间夹角的余弦.

\section{2 水连续性方程}

根据质量守恒原理, 在 $\mathrm{d} t$ 时段内流入某一物体 的水量应等于其内部储水量的增加. 设定水的渗流 可以用达西定律来描述，则对于孔隙介质有

$$
\begin{aligned}
& -\nabla^{\mathrm{T}}\left\{\boldsymbol{k}_{1} \frac{k_{r w 1}}{\mu_{w}} \nabla\left(p_{w 1}+\gamma_{w} z\right)\right\}+\frac{\bar{\alpha} \boldsymbol{k}_{1} k_{r w 1}}{\mu_{w}}\left(p_{w 1}-p_{w 2}\right) \\
& +A_{1} \frac{\partial \boldsymbol{\varepsilon}}{\partial t}+B_{1} \frac{\partial p_{w 1}}{\partial t}+E_{1} \frac{\partial p_{w 2}}{\partial t} \\
& +F_{1} \frac{\partial T}{\partial t}-\nabla^{\mathrm{T}} D_{t 1} \nabla T=0,
\end{aligned}
$$

这里, $\boldsymbol{k}_{1}, k_{r w 1}$ 分别是孔隙岩体的固有渗透率张量和比 渗透率; $\rho_{w}, \mu_{w}, \gamma_{w}$ 依次为水的密度、粘滞系数和容重; $z$ 为位置水头; $\bar{\alpha}$ 为取决于裂隙的开度与几何形状的 参数; $D_{t 1}$ 为孔隙岩体温度梯度水分扩散系数.

并且有

$$
\begin{aligned}
A_{1}= & s_{w 1}\left(\boldsymbol{m}^{\mathrm{T}}-\frac{\boldsymbol{m}^{\mathrm{T}} \boldsymbol{D}_{1}}{3 K_{s}}\right) \boldsymbol{C}_{1} \boldsymbol{D} \\
B_{1}= & D_{s 1} \varphi_{1}+\varphi_{1} \frac{s_{w 1}}{K_{w}}+s_{w 1}\left(\frac{1-\varphi_{1}}{K_{s}}-\frac{1}{\left(3 K_{s}\right)^{2}} \boldsymbol{m}^{\mathrm{T}} \boldsymbol{D}_{1} \boldsymbol{m}\right)\left(s_{w 1}\right. \\
& \left.+D_{s 1} p_{w 1}\right)-s_{w 1}\left(\boldsymbol{m}^{\mathrm{T}}-\frac{\boldsymbol{m}^{\mathrm{T}} \boldsymbol{D}_{1}}{3 K_{s}}\right)\left(s_{w 1}+D_{s 1} p_{w 1}\right) \\
& \times\left[\boldsymbol{C}_{1} \boldsymbol{D}\left(\boldsymbol{C}_{1} \boldsymbol{m}-\frac{1}{3 K_{s}} \boldsymbol{m}\right)+\left(\frac{1}{3 K_{s}} \boldsymbol{m}-\boldsymbol{C}_{1} \boldsymbol{m}\right)\right] \\
E_{1}= & -S_{w 1}\left(\boldsymbol{m}^{\mathrm{T}}-\frac{\boldsymbol{m}^{\mathrm{T}} \boldsymbol{D}_{1}}{3 K_{s}}\right) \boldsymbol{C}_{1} \boldsymbol{D}\left(s_{w 2}\right.
\end{aligned}
$$

$$
\begin{aligned}
+ & \left.D_{s 2} p_{w 2}\right)\left(\boldsymbol{C}_{2} \boldsymbol{m}-\frac{1}{3 K_{s}} \boldsymbol{m}\right), \\
F_{1}= & -\varphi_{1} s_{w 1} \beta_{w}+s_{w 1}\left[\left(\varphi_{1}-1\right) \beta_{s}+\frac{1}{3 K_{s}} \boldsymbol{m}^{\mathrm{T}} \boldsymbol{D}_{1} \boldsymbol{m} \frac{\beta_{s}}{3}\right] \\
& +\frac{1}{3} s_{w 1}\left(\boldsymbol{m}^{\mathrm{T}}-\frac{\boldsymbol{m}^{\mathrm{T}} \boldsymbol{D}_{1}}{3 K_{s}}\right)\left(\beta_{s} \boldsymbol{m}-\boldsymbol{C}_{1} \boldsymbol{D} \boldsymbol{m} \beta_{S}\right),
\end{aligned}
$$

式中, $\varphi_{1}, \beta_{s}$ 分别为孔隙岩体的孔隙率和热膨胀系数; $K_{w}$ 和 $\beta_{w}$ 分别是水的体积模量与热膨胀系数; $\boldsymbol{D}_{1}$ 是孔 隙岩体的弹性矩阵.

对于裂隙介质, 经过与上述类似的推导, 可得出 水的连续性方程为

$$
\begin{gathered}
-\nabla^{\mathrm{T}}\left\{\boldsymbol{k}_{2} \frac{k_{r w 2}}{\mu_{w}} \nabla\left(p_{w 2}+\gamma_{w} z\right)\right\}-\frac{\bar{\alpha} \boldsymbol{k}_{1} k_{r w 1}}{\mu_{w}}\left(p_{w 1}-p_{w 2}\right)+A_{2} \frac{\partial \boldsymbol{\varepsilon}}{\partial t} \\
+B_{2} \frac{\partial p_{w 2}}{\partial t}+E_{2} \frac{\partial p_{w 1}}{\partial t}+F_{2} \frac{\partial T}{\partial t}-\nabla^{\mathrm{T}} D_{t 2} \nabla T=0,
\end{gathered}
$$

式中, $\boldsymbol{k}_{2}, \boldsymbol{k}_{r w 2}$ 分别是裂隙介质的固有渗透率张量和比 渗透率; $A_{2}, B_{2}, E_{2}, F_{2}$ 可由 $A_{1}, B_{1}, E_{1}, F_{1}$ 表达式中的下 标“1”, “2”分别改为“2”, “ 1 ”得到.

在本模型中，不考虑应力场对裂隙作用所引起 的裂隙渗透张量的变化.

\section{3 能量守恒方程}

根据能量守恒原理，在 $\mathrm{d} t$ 时段内流入某一物体 的热量应等于其内能的增加. 这里认为双重介质中 温度场是单一的, 可得

$$
\begin{aligned}
& -\nabla^{\mathrm{T}} \lambda \nabla T+\left(s_{w 1} \varphi_{1} V_{1}^{a}+s_{w 2} \varphi_{2} V_{2}^{a}\right) \rho_{w} C_{w}\left(\nabla^{\mathrm{T}} T\right) \\
& +\left[\left(1-\varphi_{1}\right) C_{s} T \frac{\rho_{s}}{K_{s}}+\varphi_{1} C_{w} T \frac{\rho_{w}}{K_{w}}\right]\left(s_{w 1}+D_{s 1} p_{w 1}\right) \\
& \times \frac{\partial p_{w 1}}{\partial t}-\left\{\left(1-\varphi_{1}\right) C_{s} T \rho_{s} \beta_{s}+\left(\varphi_{1}+\varphi_{2}\right) C_{w} T \rho_{w} \beta_{w}\right. \\
& \left.-\left[\left(1-\varphi_{1}\right) \rho_{s} C_{s}+\left(\varphi_{1}+\varphi_{2}\right) \rho_{w} C_{w}\right]\right\} \frac{\partial T}{\partial t} \\
& +\frac{1}{2}\left(1-\varphi_{1}\right) \beta_{s} T \frac{\partial}{\partial t}\left(u_{i, j}+u_{j, i}\right) \delta_{i j}=0,
\end{aligned}
$$


这里, $C_{w}, C_{s}$ 分别是水及双重介质的比热; $\rho_{s}, \lambda$ 分别是 双重介质的密度和导热系数矩阵; $\boldsymbol{V}_{1}^{a}, \boldsymbol{V}_{2}^{a}$ 分别是孔隙 水和裂隙水的平均表观流速; $u_{i}, u_{j}$ 是位移分量; $\delta_{i j}$ 为 克罗内克符号.

\section{4 渗透迁移方程}

对参考文献[15]中渗透迁移方程进行了改进, 其 新意在于增加了孔隙岩体和裂隙介质之间因浓度差 产生的溶质交换, 其形式如下

$$
\begin{aligned}
R_{i} \theta_{i} \rho_{w} \frac{\partial c_{i}}{\partial t}= & \nabla^{\mathrm{T}} \theta_{i} \rho_{w} \boldsymbol{D}_{i} \nabla c_{i}-\theta_{i} \rho_{w} \boldsymbol{V}_{i} \nabla c_{i}-R_{i} \theta_{i} \rho_{w} \chi c_{i} \\
& +(-1)^{i+1} \bar{\omega} \theta_{i} \rho_{w} \boldsymbol{D}_{1}\left(c_{1}-c_{2}\right)-Q_{c i},
\end{aligned}
$$

式中, $i=1,2$ 分别对应于孔隙岩体和裂隙介质; $R_{i}=\frac{V_{i}}{V_{i}^{*}}=\left(1+\frac{\rho_{d i}}{\theta_{i}} K_{d i}\right)$, 其为阻滞系数; $V_{i}$ 为地下水 流表观速度; $V_{i}^{*}$ 为溶质输运速度; $\rho_{d i}$ 为孔隙岩体或裂 隙介质的干密度; $K_{d i}$ 为对饱和介质的分配系数; $\theta_{i}$ 为 体积含水量; $\rho_{w}$ 为流体密度; $\boldsymbol{D}_{i}$ 为扩散张量; $c_{i}$ 为溶质 浓度; $\boldsymbol{V}_{i}$ 为地下水表观流速矢量; $\chi$ 为衰减常数; $\bar{\omega}$ 为 取决于裂隙的开度与几何形状的参数; $Q_{c i}$ 为源汇项.

而扩散张量可表示为

$$
D_{i \alpha \beta}=\alpha_{i T}\left|V_{i}\right| \delta_{\alpha \beta}+\left(\alpha_{i L}-\alpha_{i T}\right) \frac{V_{i \alpha} V_{i \beta}}{\left|V_{i}\right|}+\alpha_{i m} \tau_{i} \delta_{\alpha \beta},
$$

式中, $\alpha_{i T}$ 为横向弥散度; $\alpha_{i L}$ 为纵向弥散度; $\left|V_{i}\right|$ 为表观 流速的绝对值; $\alpha_{i m}$ 为分子扩散系数; $\tau_{i}$ 为曲折率; $\delta_{\alpha \beta}$ 为克罗内克符号.

在上述式子中, (1), (7) (10)式即是热-水-应力-迁 移耦合控制方程.

\section{3 有限元格式}

\section{1 空间域离散}

使用 Galerkin 方法, 对前述的应力平衡方程、水 连续性方程、能量守恒方程和渗透迁移方程进行空间 域离散, 整理可得

$$
\boldsymbol{K} \frac{\mathrm{d} \overline{\boldsymbol{u}}}{\mathrm{d} t}+\boldsymbol{C} \frac{\mathrm{d} \bar{p}_{w 1}}{\mathrm{~d} t}+\boldsymbol{I} \frac{\mathrm{d} \bar{p}_{w 2}}{\mathrm{~d} t}+\boldsymbol{J} \frac{\mathrm{d} \bar{T}}{\mathrm{~d} t}=\frac{\mathrm{d} \boldsymbol{f}}{\mathrm{d} t},
$$

$\boldsymbol{E}_{1} \frac{\mathrm{d} \overline{\boldsymbol{u}}}{\mathrm{d} t}+\boldsymbol{G}_{1} \frac{\mathrm{d} \bar{p}_{w 1}}{\mathrm{~d} t}+\boldsymbol{H}_{1} \frac{\mathrm{d} \bar{p}_{w 2}}{\mathrm{~d} t}+\boldsymbol{F}_{1} \bar{p}_{w 1}+\boldsymbol{M} \bar{p}_{w 1}-\boldsymbol{M} \bar{p}_{w 2}$

$+\boldsymbol{U}_{1} \bar{T}+\boldsymbol{V}_{1} \frac{\mathrm{d} \bar{T}}{\mathrm{~d} t}=\mathbf{0}$,

$\boldsymbol{E}_{2} \frac{\mathrm{d} \overline{\boldsymbol{u}}}{\mathrm{d} t}+\boldsymbol{G}_{2} \frac{\mathrm{d} \bar{p}_{w 1}}{\mathrm{~d} t}+\boldsymbol{H}_{2} \frac{\mathrm{d} \bar{p}_{w 2}}{\mathrm{~d} t}+\boldsymbol{F}_{2} \bar{p}_{w 2}-\boldsymbol{M} \bar{p}_{w 1}+\boldsymbol{M} \bar{p}_{w 2}$

$+\boldsymbol{U}_{2} \bar{T}+\boldsymbol{V}_{2} \frac{\mathrm{d} \bar{T}}{\mathrm{~d} t}=\boldsymbol{X}$,

$\boldsymbol{A} \frac{\mathrm{d} \overline{\boldsymbol{u}}}{\mathrm{d} t}+\boldsymbol{P} \frac{\mathrm{d} \bar{p}_{w 1}}{\mathrm{~d} t}+\boldsymbol{R} \bar{T}+\boldsymbol{Q} \frac{\mathrm{d} \bar{T}}{\mathrm{~d} t}=\boldsymbol{Y}$,

$\boldsymbol{W}_{1} \bar{c}_{1}+\boldsymbol{L}_{1} \frac{\mathrm{d} \bar{c}_{1}}{\mathrm{~d} t}+\boldsymbol{S} \bar{c}_{1}-\boldsymbol{S} \bar{c}_{2}=\boldsymbol{Z}$,

$\boldsymbol{W}_{2} \bar{c}_{2}+\boldsymbol{L}_{2} \frac{\mathrm{d} \bar{c}_{2}}{\mathrm{~d} t}+\boldsymbol{S} \bar{c}_{2}-\boldsymbol{S} \bar{c}_{1}=\mathbf{0}$,

上述式子中, 未知量为 $\bar{u}, \bar{p}_{w 1}, \bar{p}_{w 2}, \bar{T}, \bar{c}_{1}, \bar{c}_{2}$, 其前面的黑体大写字母为常数矩阵; $\boldsymbol{f}$ 为取决于作用 在单元上的体力、面力与初应力的力荷载; $\boldsymbol{X}, \boldsymbol{Y}, \boldsymbol{Z}$ 分 别为由源汇项和边界流量项求得的水荷载、热荷载和 溶质浓度“荷载”.

\section{2 时间域离散}

认为在每个时步 $\Delta t$ 内 $\overline{\boldsymbol{u}}, \bar{p}_{w 1}, \bar{p}_{w 2}, \bar{T}, \bar{c}_{1}, \bar{c}_{2}$ 是 线性变化的, 故可将(12)式变为如下的迭代求解方程

$$
\begin{aligned}
& {\left[\begin{array}{cccc}
\boldsymbol{K} & \boldsymbol{G} & \boldsymbol{I} & \boldsymbol{J} \\
\boldsymbol{E}_{1} & \boldsymbol{G}_{1}+\eta\left(\boldsymbol{F}_{1}+\boldsymbol{M}\right) \Delta t_{k} & \boldsymbol{H}_{1}-\eta \boldsymbol{M} \Delta t_{k} & \boldsymbol{V}_{1}+\eta \boldsymbol{U}_{1} \Delta t_{k} \\
\boldsymbol{E}_{2} & \boldsymbol{G}_{2}-\eta \boldsymbol{M} \Delta t_{k} & \boldsymbol{H}_{2}+\eta\left(\boldsymbol{F}_{2}+\boldsymbol{M}\right) \Delta t_{k} & \boldsymbol{V}_{2}+\eta \boldsymbol{U}_{2} \Delta t_{k} \\
\boldsymbol{A} & \boldsymbol{P} & \boldsymbol{0} & \boldsymbol{Q}+\eta \boldsymbol{R} \Delta t_{k}
\end{array}\right]_{t, \eta}\left\{\begin{array}{c}
\overline{\boldsymbol{U}} \\
\bar{P}_{w 1} \\
\bar{P}_{w 2} \\
\bar{T}
\end{array}\right\}_{t_{k}+\Delta t_{k}}} \\
& =\left[\begin{array}{cccc}
\boldsymbol{K} & \boldsymbol{G} & \boldsymbol{I} & \boldsymbol{J} \\
\boldsymbol{E}_{1} & \boldsymbol{G}_{1}-(1-\eta)\left(\boldsymbol{F}_{1}+\boldsymbol{M}\right) \Delta t_{k} & \boldsymbol{H}_{1}+(1-\eta) \boldsymbol{M} \Delta t_{k} & \boldsymbol{V}_{1}-(1-\eta) \boldsymbol{U}_{1} \Delta t_{k} \\
\boldsymbol{E}_{2} & \boldsymbol{G}_{2}+(1+\eta) \boldsymbol{M} \Delta t_{k} & \boldsymbol{H}_{2}-(1-\eta)\left(\boldsymbol{F}_{2}+\boldsymbol{M}\right) \Delta t_{k} & \boldsymbol{V}_{2}-(1-\eta) \boldsymbol{U}_{2} \Delta t_{k} \\
\boldsymbol{A} & \boldsymbol{P} & \mathbf{0} & \boldsymbol{Q}-(1-\eta) \boldsymbol{R} \Delta t_{k}
\end{array}\right]_{t, \eta}\left\{\begin{array}{c}
\overline{\boldsymbol{U}} \\
\bar{P}_{w 1} \\
\bar{P}_{w 2} \\
\bar{T}
\end{array}\right\}_{t_{k}}+\left\{\begin{array}{c}
\frac{\partial \boldsymbol{f}}{\partial t} \\
0 \\
X \\
Y
\end{array}\right\}_{t, \eta} \Delta t_{k},
\end{aligned}
$$




$$
\begin{aligned}
& {\left[\begin{array}{cc}
\boldsymbol{L}_{1}+\eta\left(\boldsymbol{W}_{1}+\boldsymbol{S}\right) \Delta t_{k} & -\eta \boldsymbol{S} \Delta t_{k} \\
-\eta \boldsymbol{S} \Delta t_{k} & \boldsymbol{L}_{2}+\eta\left(\boldsymbol{W}_{2}+\boldsymbol{S}\right) \Delta t_{k}
\end{array}\right]_{t, \eta}\left\{\begin{array}{l}
\bar{c}_{1} \\
\bar{c}_{2}
\end{array}\right\}_{t_{k}+\Delta t_{k}}} \\
& =\left[\begin{array}{cc}
\boldsymbol{L}_{1}-(1-\eta)\left(\boldsymbol{W}_{1}+\boldsymbol{S}\right) \Delta t_{k} & (1+\eta) \boldsymbol{S} \Delta t_{k} \\
(1+\eta) \boldsymbol{S} \Delta t_{k} & \boldsymbol{L}_{2}-(1-\eta)\left(\boldsymbol{W}_{2}+\boldsymbol{S}\right) \Delta t_{k}
\end{array}\right]_{t, \eta}\left\{\begin{array}{l}
\bar{c}_{1} \\
\bar{c}_{2}
\end{array}\right\}_{t_{k}}+\left\{\begin{array}{l}
Z \\
0
\end{array}\right\}_{t, \eta} \Delta t_{k} .
\end{aligned}
$$

在每一时步的迭代计算中, 若前后两次迭代所 得的孔隙水压力、裂隙水压力和相应的渗透系数的相 对误差均小于 0.01 , 则转入下一时步的迭代, 直到完 成整个确定时段的分析.

\section{4 双重孔隙-裂隙介质耦合模型的验证}

对于上述的双重孔隙-裂隙介质热-水-应力-迁移 耦合模型, 笔者开发了相应的三维有限元程序. 耦合 模型及有限元程序的合理性验证如下.

由于目前尚无热-水-应力-迁移耦合模型的解析 解可供对比, 加之一时也难以找到合适的现场试验 数据来模拟, 故笔者试图用已有的数值解来验证本 模型. Elsworth 曾提出过一个双重孔隙介质的水-应力 耦合模型. 作为考题, 其假定:一饱和柱状体中分布 有间距为 $0.1 \mathrm{~m}$ 的正交裂隙, 在轴向承受均布荷载 $P_{0}$, 材料的物理力学参数见表 1 . 从而使用相应的有限元 程序对该柱状体进行了流-固耦合数值计算模拟, 其 结果符合客观规律 ${ }^{[16]}$. 笔者为了将所建立的双重孔 隙-裂隙介质热-水-应力-迁移耦合模型与 Elsworth 的 成果相比较, 也针对上述柱状体进行了流-固耦合(温 度为常量, 暂不计迁移)的有限元分析. Elsworth 和笔 者得出的柱状体中孔隙流体压力和端面沉降的分布 及随时间变化的曲线见图 3 和 4. 看到 2 种程序的模 拟结果相当接近, 这表明了笔者所建立的模型是可 靠的.

\section{Table 1 Column coefficients}

\begin{tabular}{lc}
\hline Young's modulus, $E(\mathrm{MPa})$ & 1.0 \\
Poisson's ratio, $\mu$ & 0.15 \\
Fissure stiffness, $k_{n}(\mathrm{MPa} / \mathrm{m})$ & 0.1 \\
Fluid bulk modulus, $k_{f}(\mathrm{MPa})$ & 0.1 \\
Matrix porosity, $\phi_{1}$ & 0.1 \\
Fissure porosity, $\phi_{2}$ & 0.05 \\
Matrix permeability, $k_{1} / \mu\left(\mathrm{m}^{2} / \mathrm{Pa} \cdot \mathrm{s}\right)$ & $0.01 \times 10^{-9}$ \\
Fissure permeability, $k_{2} / \mu\left(\mathrm{m}^{2} / \mathrm{Pa} \cdot \mathrm{s}\right)$ & $0.1 \times 10^{-6}$ \\
Fissure spacing, $s(\mathrm{~m})$ & 0.1 \\
\hline
\end{tabular}

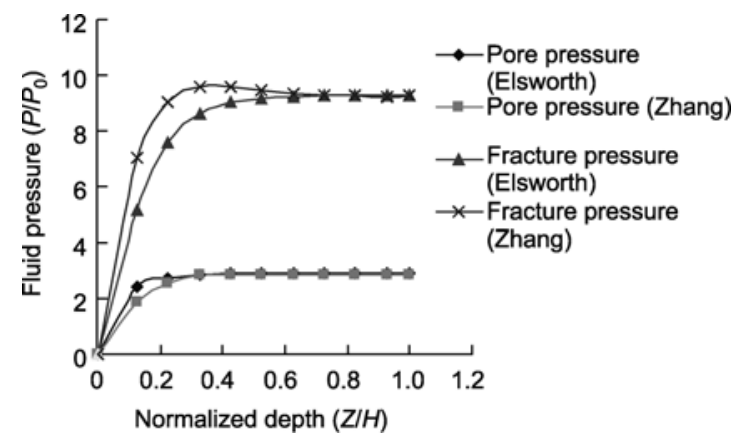

图 $34000 \mathrm{~s}$ 时柱状体中孔隙流体压力分布

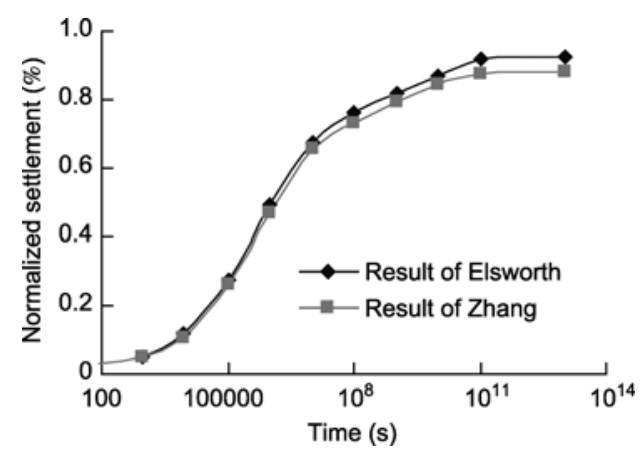

图 4 柱状体表面沉降-时间曲线

\section{5 算例}

根据目前国外所设计的核废物地质处置概念库 的构成 ${ }^{[14]}$, 立足于方法论的定性研究, 笔者采用了一 个简单的计算模型, 见图 5 . 该模型的尺寸为长 $\times$ 宽 $\times$ 高=10 $\mathrm{m} \times 10 \mathrm{~m} \times 20 \mathrm{~m}$, 其内部缓冲材料的尺寸为长 $\times$ 宽 $\times$ 高 $=4 \mathrm{~m} \times 4 \mathrm{~m} \times 4 \mathrm{~m}$, 核废物咜存罐位于模型的中心. 由于对称性, 取沿垂向剖开的 $1 / 4$ 模型作为分析域, 对应的有限元网格见图 6, 其中有 4891 个节点, 4000 个八节点等参数单元. 假定核废物咜存罐的埋深为 $500 \mathrm{~m}$, 岩体中的初始温度为 $20.0^{\circ} \mathrm{C}$. 取边界条件为: 顶、底面水头及温度固定，其余面为绝热及不透水面， 四个垂直面及底面的法线方向位移约束, 而顶面是 位移自由的, 其上并作用有上部岩体自重转化的节 点荷载. 岩体是非饱和介质, 其中发育有一组水平裂 隙及两组正交的垂直裂陌. 笔者参考了美国 DST 试 

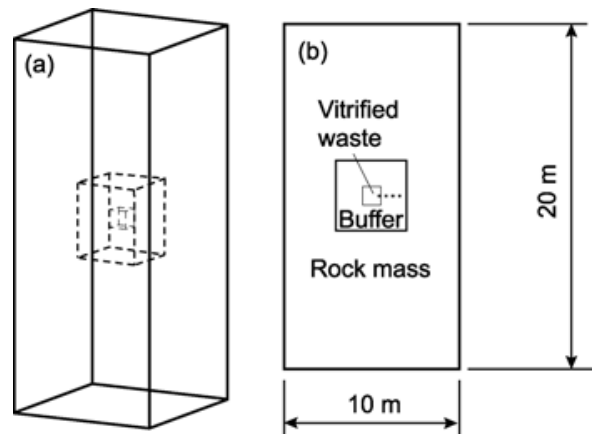

图 5 核废物地质处置模型

(a) 立体图; (b) 剖面图(其中点号由内向外依次 为 $2422,2423,2424,2425$ )

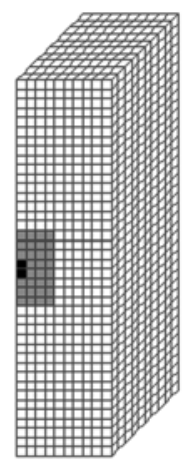

图 6 三维有限元网格
验 ${ }^{[17]}$ 和日本 BMT1 试验 ${ }^{[14]}$ 的情况, 取岩石、缓冲层、 固化体和裂隙组的主要计算参数见表 2 和 3 .

孔隙岩体、裂隙介质和缓冲层的初始饱和度依次 为 $0.9,0.1$ 和 0.2 , 其水分特性曲线符合 Van Genuchten 模型, 即

$$
s_{w}=\left(s_{w s}-s_{w r}\right)\left(1+|\alpha \psi|^{n}\right)^{-m}+s_{w r},
$$

式中, $\alpha, n, m=1-1 / n$ 为材料参数; $\psi$ 为水势; $s_{w s}, s_{w r}$ 分 别为最大、最小饱和度. 对于孔隙岩体: $\alpha=2.25 \times 10^{-6}$ $\mathrm{m}^{-1}, n=1.33, s_{w s}, s_{w r}$ 分别为 1.0 和 0.18 , 从而孔隙岩 体中的初始孔隙水压力为 $-0.34 \mathrm{MPa}$; 对于裂隙介质: $\alpha=9.74 \times 10^{-5} \mathrm{~m}^{-1}, n=1.97, s_{w s}, s_{w r}$ 分别为 1.0 和 0.01 , 故裂隙介质中的初始孔隙水压力为 $-0.14 \mathrm{MPa}$; 对于 缓冲层: $\alpha=8.0 \times 10^{-3} \mathrm{~m}^{-1}, n=1.6, s_{w s}, s_{w r}$ 分别为 1.0 和 0.0 , 所以缓冲层中的初始孔隙水压力为 $-18.12 \mathrm{MPa}$.

比渗透率与饱和度的关系为

$$
k_{r w}=s_{w}^{2.0} .
$$

取孔隙岩体、裂隙介质和缓冲层的温度梯度水分 扩散系数均为

$$
D_{t}=2.0 \times 10^{-10} \mathrm{~m}^{2} / \mathrm{s}{ }^{\circ} \mathrm{C} .
$$

假定核废料咜存罐埋置完毕后，由于某种原因 伧存罐有所损坏，并以恒定的浓度及速率均匀地向 近场泄漏放射性核素，以此时为计算起点，咜存罐

Table 2 Main computation parameters for rock, buffer and vitrified waste

\begin{tabular}{lccc}
\hline \multicolumn{1}{c}{ Property } & Rock mass & Buffer & Vitrified waste \\
\hline Density, $\rho\left(\mathrm{kN} / \mathrm{m}^{3}\right)$ & 25.3 & 16.0 & 25.0 \\
Porosity, $\phi_{1}$ & 0.11 & 0.33 & 0.0 \\
Permeability, $k_{1} / \mu_{w}\left(\mathrm{~m}^{2} / \mathrm{Pa} . \mathrm{s}\right)$ & $1.24 \times 10^{-10}$ & $4.0 \times 10^{-13}$ & $1.0 \times 10^{-27}$ \\
Young's modulus, $E(\mathrm{MPa})$ & $1.48 \times 10^{4}$ & $5.0 \times 10^{2}$ & $5.3 \times 10^{4}$ \\
Poisson's ratio, $\mu$ & 0.21 & 0.3 & 0.21 \\
Specific heat, $C\left(\mathrm{~kJ} / \mathrm{kg}^{\circ} \mathrm{C}\right)$ & 0.95 & 0.34 & 0.7 \\
Thermal expan. coeff., $\beta\left(1 /{ }^{\circ} \mathrm{C}\right)$ & $8.8 \times 10^{-6}$ & $1.0 \times 10^{-6}$ & $1.0 \times 10^{-5}$ \\
Thermal conductivity, $\lambda\left(\mathrm{W} / \mathrm{m}^{\circ} \mathrm{C}\right)$ & 2.0 & 0.44 & 5.3 \\
\hline
\end{tabular}

Table 3 Parameters for fracture sets used in calculation

\begin{tabular}{lcc}
\hline \multicolumn{1}{c}{ Parameter } & Horizontal fracture & Vertical fracture \\
\hline Spacing, $S(\mathrm{~m})$ & 0.3 & 0.3 \\
Continuity ratio, $A$ & 1 & 1 \\
Dip angle, $\theta\left(^{\circ}\right)$ & 0 & 90 \\
Normal stiffness, $k_{n}(\mathrm{MPa} / \mathrm{m})$ & 1000.0 & 2000.0 \\
Shearing stiffness, $k_{s}(\mathrm{MPa} / \mathrm{m})$ & 500.0 & 0.01 \\
Porosity, $\phi_{2}$ & 1000.0 \\
Constant, $\bar{\alpha}\left(\mathrm{m}^{-2}\right)$ & 100.0 & 0.01 \\
Permeability, $k_{2} / \mu_{w}\left(\mathrm{~m}^{2} / \mathrm{Pa} \cdot \mathrm{s}\right)$ & $1.0 \times 10^{-6}$ & 100.0 \\
\hline
\end{tabular}

Note: the properties of two vertical fracture sets are the same, and the shearing stiffnesses in any direction on a fracture surface are the same. 
为孔隙岩体中的源项, 某种放射性核素的释放强度 为 $Q_{c}=1.44 \times 10^{-6} \mathrm{~mol} \cdot \mathrm{kg} / \mathrm{m}^{3} \cdot \mathrm{s}^{-1}$. 设与核素的渗透 迁移有关且在计算中不变的参数为: 孔隙曲折率 $\tau_{1}$, $\tau_{2}$ 分别为 $0.4,0.8$, 纵向弥散度 $\alpha_{1 L}, \alpha_{2 L}$ 分别为 1.0 和 $2.0 \mathrm{~m}$, 横向弥散度 $\alpha_{i T}=\alpha_{i L} / 10$, 分子扩散系数 $\alpha_{1 m}$, $\alpha_{2 m}$ 分别为 $1.0 \times 10^{-9}$ 和 $2.0 \times 10^{-9} \mathrm{~m}^{2} / \mathrm{s}$, 分配系数 $K_{d 1}$, $K_{d 2}$ 分别为 8.0 和 $5.3 \mathrm{~mL} / \mathrm{g}$, 干密度 $\rho_{d 1}, \rho_{d 2}$ 分别为 23.0 和 $21.0 \mathrm{~kg} / \mathrm{m}^{3}$, 参数 $\bar{\omega}$ 为 $100.0 \mathrm{~m}^{-2}$, 放射性核素的衰 减常数 $\chi=\ln 2 / T_{\text {half }}$, 其中 $T_{\text {half }}$ 是半衰期, 取为 $1000 \mathrm{a}$. 核废物以 $300 \mathrm{~W}$ 的不变功率释放热量, 取时间步长为 $100000 \mathrm{~s}$, 经历了 $4 \mathrm{a}$. 计算的主要结果及分析如下.

图 5(b)显示的剖面即为有限元网格的正面, 上面 标注了 4 个计算结果输出点的位置, 其中 2422 点位 于贮存罐的外缘, 2423 点、2424 点位于缓冲层的内部, 2425 点位于缓冲层和处置孔壁的交界处. 图 7 是上述 4 个点的温度随时间变化曲线, 从中看到, 各点的温 度在开始阶段快速上升, 离贮存罐越近的点其温度 越高, 在前 $0.1 \mathrm{a}$ 温度增加最明显, 之后温度增长缓 慢, 到 $4 \mathrm{a}$ 时 4 个点的温度依次为 $72.5^{\circ} \mathrm{C}(2422$ 点)、 $45.0^{\circ} \mathrm{C}$ (2423 点)、 $32.3^{\circ} \mathrm{C}\left(2424\right.$ 点)和 $24.8^{\circ} \mathrm{C}(2425$ 点). 图 8 是 $4 \mathrm{a}$ 时在模型高度之半的水平剖面上贮存罐周 围 $2.5 \mathrm{~m} \times 2.5 \mathrm{~m}$ 范围内的温度等值线分布.

图 9 是 2422,2423,2424 和 2425 各点处的孔隙水 压力随时间的变化曲线. 从中得知, 2425 点位于缓冲 层和处置孔壁的交界处, 该点得到地下水的快速浸 润, 其负孔隙水压力在约 0.46 年时降到了 $-0.25 \mathrm{MPa}$. 而位于缓冲层内部的 3 个点的负孔隙水压力开始均 有所上升, 其原因在于: 一是在开始阶段热-水-应力 耦合现象强烈, 缓冲材料受到围岩的挤压作用后其 含水量减少(特别是在离围岩较近之处, 如 2424 点), 二是在开始阶段缓冲层中距离拒存罐近的部位的温 度升高较快, 温度梯度引起了水分扩散(向外), 而水

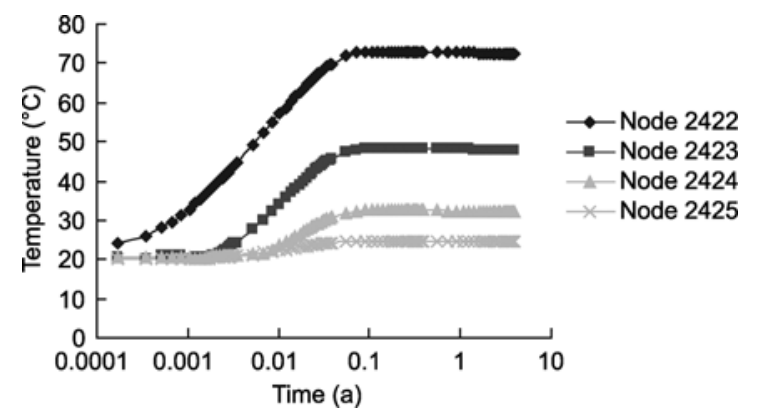

图 7 温度-时间曲线

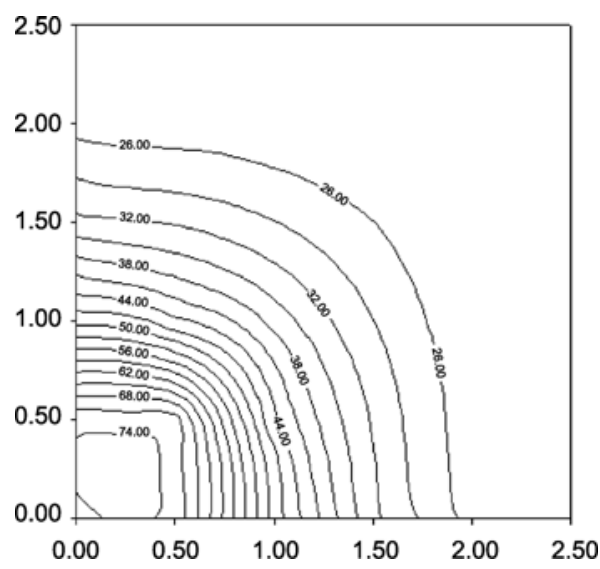

图 $84 \mathrm{a}$ 时缓冲层水平剖面上温度等值线 $\left({ }^{\circ} \mathrm{C}\right)$

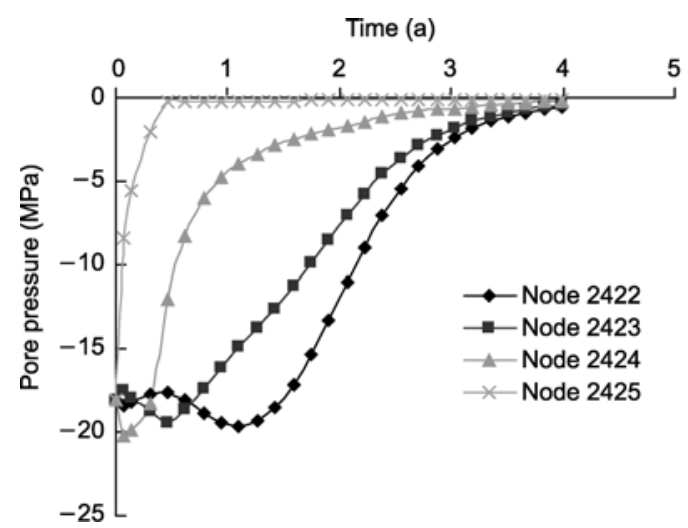

图 9 缓冲层中孔隙水压力-时间曲线

力梯度产生的水分迁移(向内)尚未到达该部位, 使得 该部位的干燥程度不断增加. 一定时间后随着地下 水分的到达和浸润作用的增强, 缓冲层中各点的负 孔隙水压力才逐渐变小. 到 $4 \mathrm{a}$ 时 4 个点的负孔隙水 压力依次为 $-0.53 \mathrm{MPa}(2422$ 点)、 $-0.44 \mathrm{MPa}$ (2423 点)、 $-0.22 \mathrm{MPa}(2424$ 点)和 $-0.13 \mathrm{MPa}(2425$ 点).

图 10 是 $0.8 \mathrm{a}, 2.4 \mathrm{a}$ 和 $4 \mathrm{a}$ 时在模型高度之半的 水平剖面上缓冲层区域中的孔隙水压力等值线分布.

图 11 是 $0.8 \mathrm{a}$ 和 $2.4 \mathrm{a}$ 时在模型高度之半的水平 剖面上围岩区域中的孔隙水压力和裂隙水压力等值 线分布. 从中看到, 随着时间的推移, 孔隙水压力和 裂隙水压力的变化趋于平缓,其差值逐渐减小. 如在 沿水平方向距它存罐外缘 $2.0 \mathrm{~m}$ 处, 在 $0.8 \mathrm{a}$ 和 $2.4 \mathrm{a}$ 时的孔隙水压力和裂隙水压力分别为: $-0.130 \mathrm{MPa}$, $-0.128 \mathrm{MPa} ;-0.120 \mathrm{MPa},-0.121 \mathrm{MPa}$. 图 12 是 $0.8 \mathrm{a}$ 时该水平剖面上的孔隙水流速和裂隙水流速矢量分 布(后者的比例尺为前者的 1/200000). 如在沿水平方 

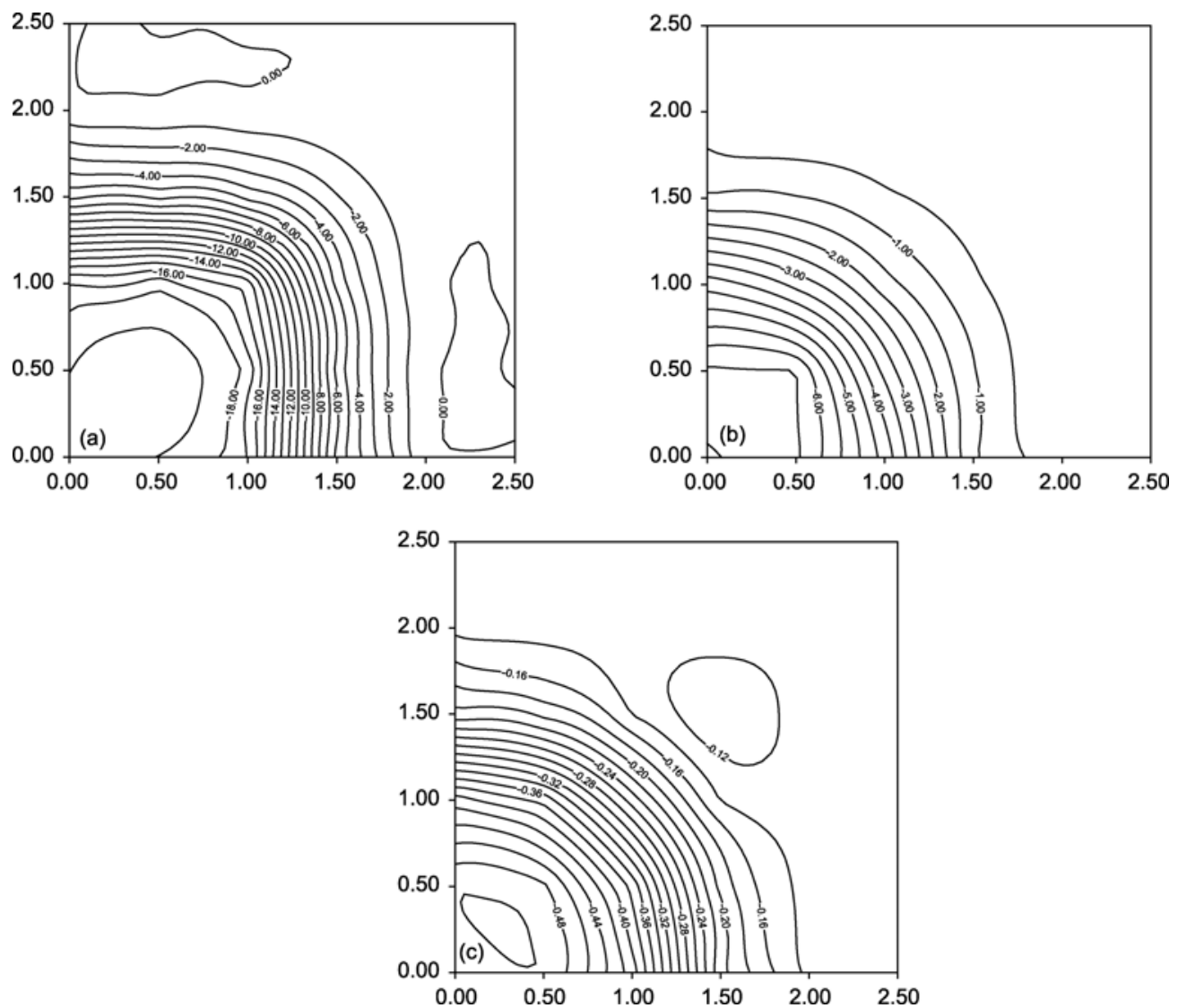

图 10 缓冲层水平剖面上孔隙水压力等值线 (MPa)

(a) $0.8 a$ 时; (b) $2.4 a$ 时; (c) $4 a$ 时

向距咜存罐外缘 2.0 和 $3.0 \mathrm{~m}$ 处, 孔隙岩体和裂隙介 质中的流速分别为 $3.77 \times 10^{-9}, 1.34 \times 10^{-3}, 5.54 \times 10^{-10}$ 和 $1.55 \times 10^{-4} \mathrm{~m} / \mathrm{s}$, 可见裂隙中的流速要比裂隙中的流 速大 6 个数量级左右.

图 13 是上述缓冲层中 4 个点处的核素浓度随时 间的变化曲线. 看到位于咜存罐外缘的 2422 点处的 核素浓度最大, 在 $4 \mathrm{a}$ 的时间内基本呈直线上升, 而 其余 3 个点处的核素浓度依点距咜存罐的近、远而逐 次降低，并且随时间显示了非线性的的变化，这表明 近场的热-水-应力耦合过程是复杂的, 核素迁移要受 其影响. 到 $4 \mathrm{a}$ 时 2422, 2423, 2424 和 2425 各点的核 素浓度依次为: $1.26,0.28,0.07$ 和 $0.06 \mathrm{~mol} / \mathrm{m}^{3}$. 图 14 是 $4 \mathrm{a}$ 时在模型高度之半的水平剖面上咜存罐周围 $2.5 \mathrm{~m} \times 2.5 \mathrm{~m}$ 范围内的核素浓度等值线分布.

在上述水平剖面上 $4 \mathrm{a}$ 时的正应力 $\sigma_{x}, \sigma_{y}$ 和 $\sigma_{z}$ 的等 值线见图 15. 从中看到, 应力分布集中的区域位于缓 冲层和处置孔壁交界两侧附近, 该面上最大的 $\sigma_{x}, \sigma_{y}$
和 $\sigma_{z}$ 值依次为 $-2.47,-2.47$ 和 $-16.6 \mathrm{MPa}$ (均为压性).

\section{6 结束语}

对于饱和-非饱和的遍有节理岩体, 建立了一种 双重孔隙-裂隙介质的热-水-应力-迁移耦合三维模型, 其中岩体的应力场和温度场是单一的, 但孔隙岩体 和裂隙介质具有不同的渗流场和浓度场, 并开发了 相应的三维有限元计算程序.

对一个假定的高放废物地质处置库进行了三维 数值模拟. 其计算结果表明: 缓冲层中温度在开初的 $0.1 \mathrm{a}$ 内快速上升, 之后变化很小, 计算终了 (4 a)时, 缓冲层内的温度可达到 $25.0 \sim 73.0^{\circ} \mathrm{C}$; 在开始阶段, 主要是因为温度梯度的作用，核废料諚存罐附近的 孔隙水分向远场迁移，导致该部分缓冲材料变得较 为 “干燥”, 从而距咜存罐较近部位的负孔隙水压力 有所上升; 又由于来自围岩的地下水的不断内浸, 当 

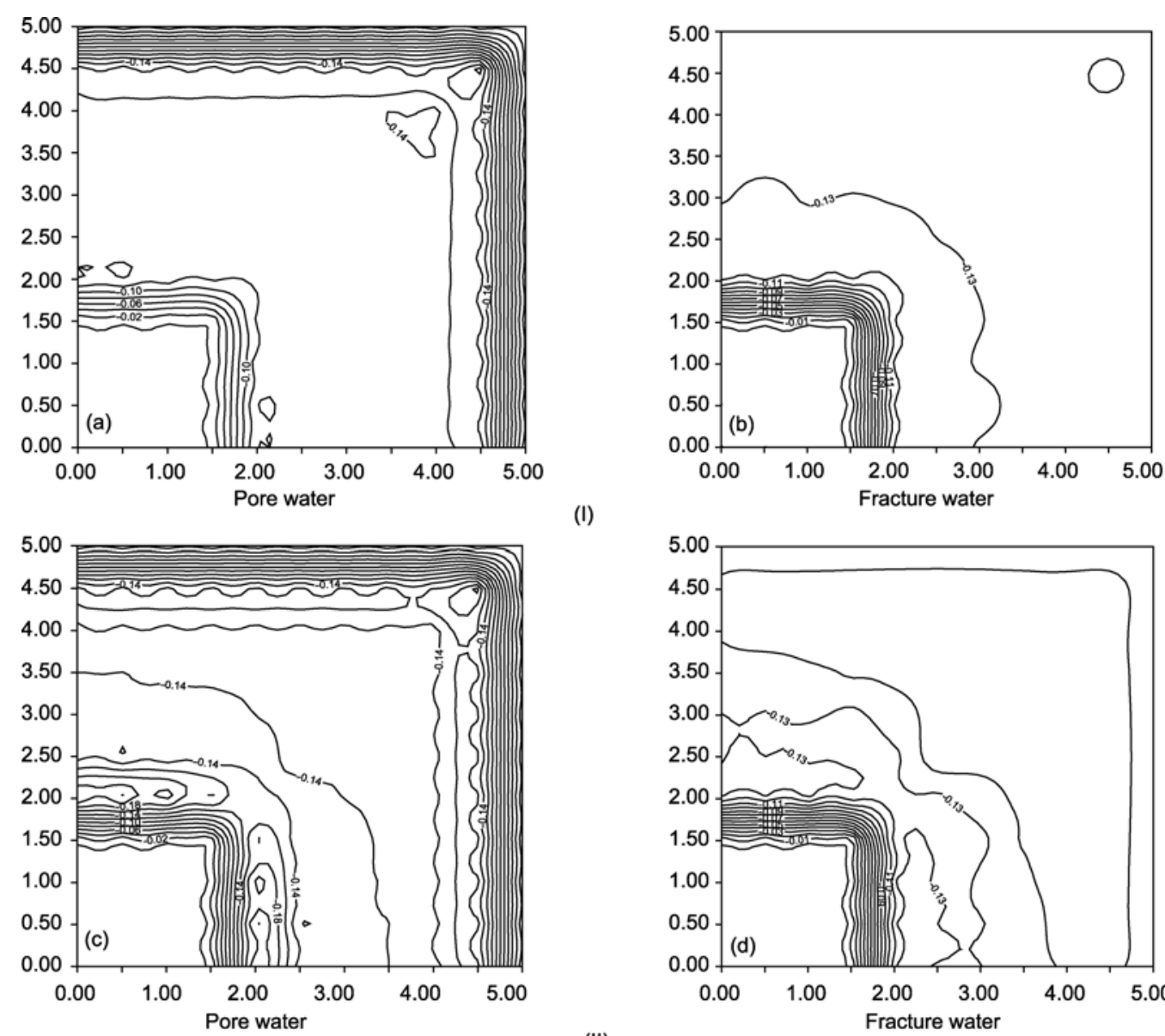

(I)

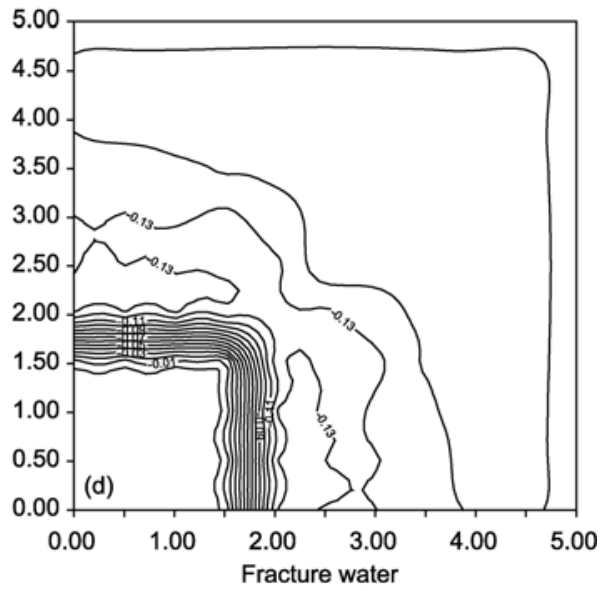

(II)

图 11 岩体水平剖面上孔隙水压力等值线 (MPa)

(a) 孔隙水; (b) 裂隙水; (c) 孔隙水; (d) 裂隙水
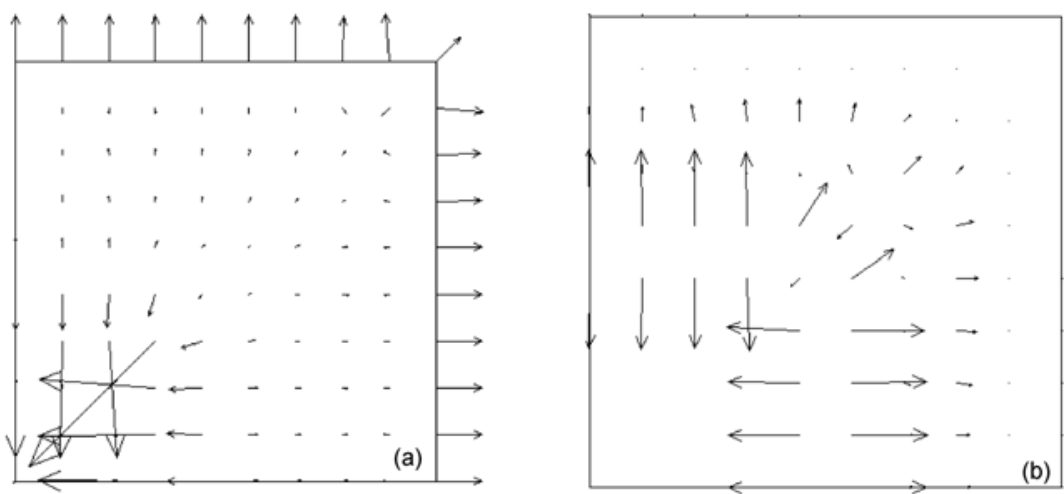

图 $120.8 \mathrm{a}$ 时时岩体水平剖面上地下水流速矢量

(a) 孔隙水; (b) 裂隙水 


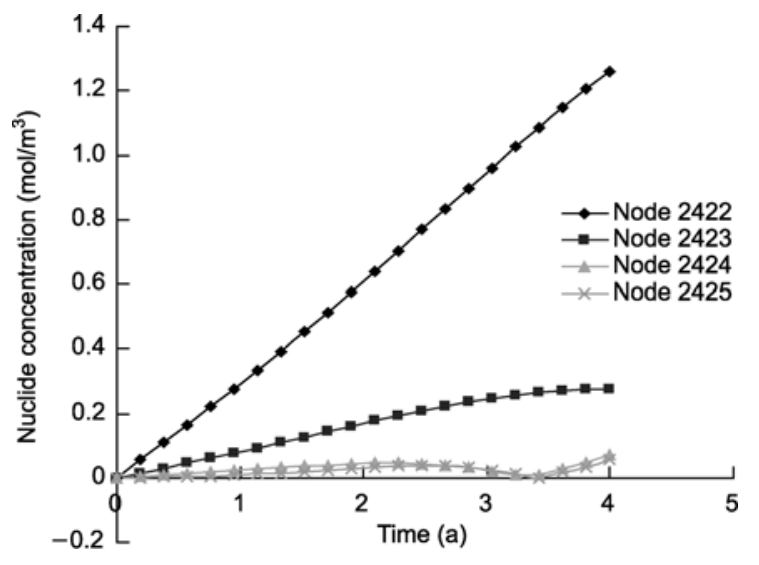

图 13 缓冲层中核素浓度-时间曲线

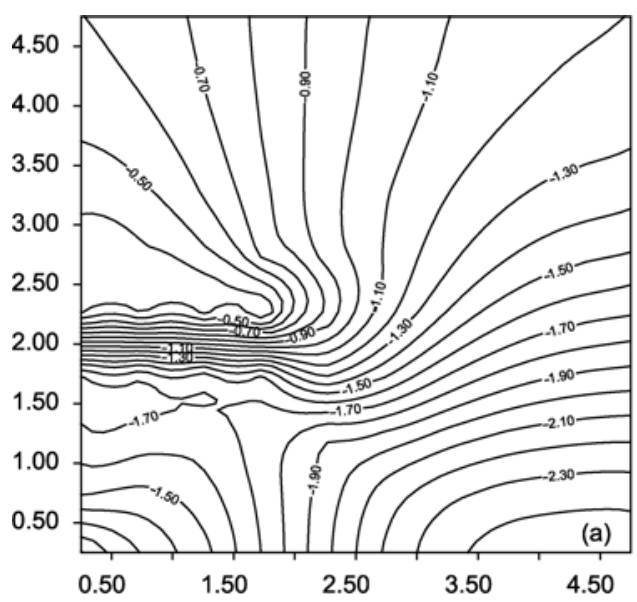

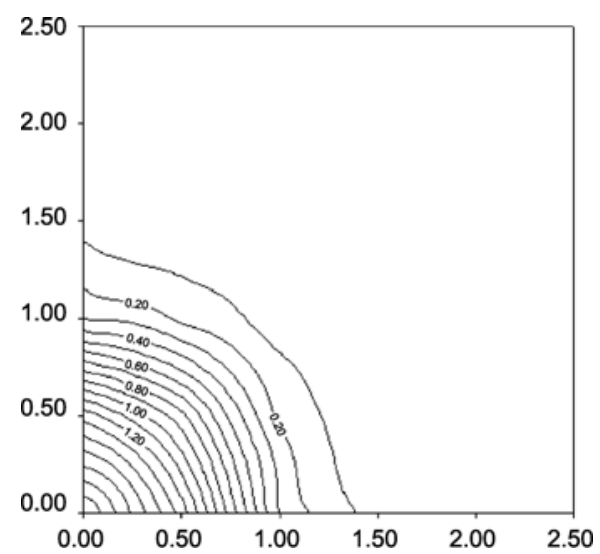

图 $144 \mathrm{a}$ 缓冲层水平剖面上核素浓度等值线 $\left(\mathrm{mol} / \mathrm{m}^{3}\right)$

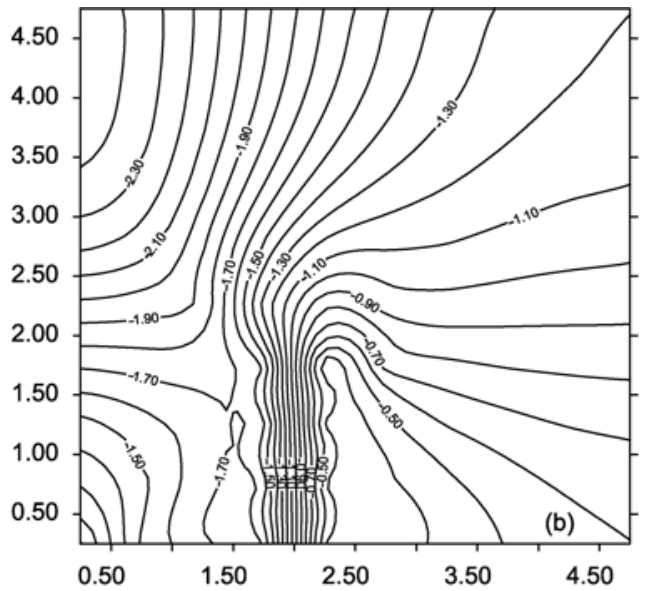

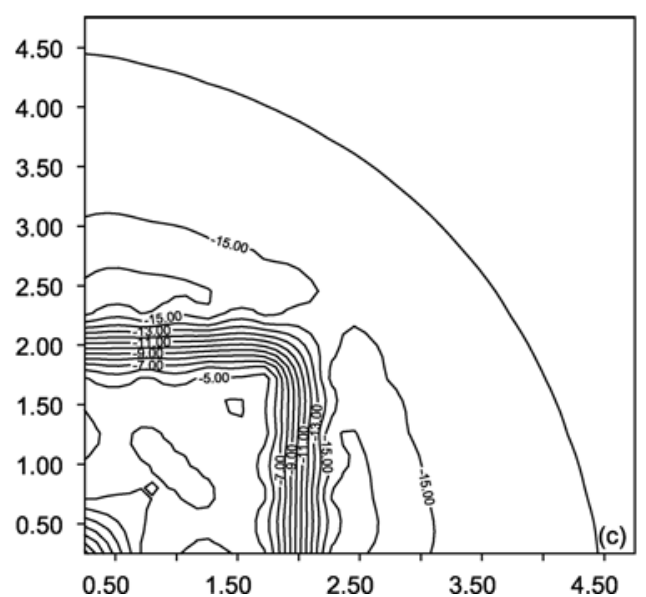

图 $154 \mathrm{a}$ 时岩体水平剖面上正应力等值线 $(\mathrm{MPa})$

(a) $\sigma_{x}$; (b) $\sigma_{y}$; (c) $\sigma_{z}$

缓冲层中因 “渗”获得的水分大于因 “热”失去的水分 时, 又使得其中的负裂隙水压力持续下降(从初值的 $-18.12 \mathrm{MPa}$ 变为终值的约 $-0.13 \sim-0.53 \mathrm{MPa}$ ); 随着时
间的推移，围岩中孔隙水压力和裂隙水压力的变化 趋于平缓, 其差值逐渐减小; 尽管围岩中孔隙水饱和 度大致是裂隙水饱和度的 9 倍, 但因裂隙的渗透系数 
比孔隙的渗透系数大 4 个数量级, 故裂隙中地下水的 流速约是孔隙中相应值的 6 倍左右; 在计算的始终, 缓冲层内咜存罐外缘点处的核素浓度最大, 并且在 4 $\mathrm{a}$ 的时间内基本呈直线上升, 而其余点处的核素浓度 随着点与拒存罐之间距离的增加而逐次降低, 以及 在时间域内显示了非线性的变化; 而处置库围岩中 应力分布集中的区域位于缓冲层和处置孔壁交界两
侧附近.

对于修建在遍有节理的双重孔隙-裂隙岩体中的 高放废物地质处置库, 由于裂隙的渗透系数和扩散 系数比孔隙的相应值大许多, 会使得水分和核素沿 裂隙较快地输运，与单一孔隙介质中的情况相比可 能更为不利于环境安全，对此应用双重孔隙-裂隙介 质的热-水-应力-迁移耦合模型进行分析较为可靠.

\section{参考文献}

1 王驹, 郑华铃, 徐国庆, 等. 中国高放废物地质处置研究十年进展. 北京: 原子能出版社, 2004. 1-12

2 TRW Environmental Safety Systems Inc. Drift scale test progress report No.1. Las Vegas: TRW Environmental Safety Systems Inc., 1998

3 赵延林, 曹平, 赵阳升, 等. 双重介质温度场-渗流场-应力场耦合模型及三维数值研究. 岩石力学与工程学报, 2007, 26: 4024一-4031

4 刘耀儒, 杨强, 黄岩松, 等. 基于双重孔隙介质模型的渗流-应力耦合并行数值分析. 岩石力学与工程学报, 2007, 26: 705-711

5 Olivella S, Gens A. Double structure THM analyses of a heating test in a fractured tuff incorporating intrinsic permeability variations. Int J Rock Mech Min Sci, 2005, 42: 667-679

6 Rutqvist J, Tsang C F. Analysis of thermal-hydrologic-mechanical behavior near an emplacement drift at Yucca mountain. J Contam Hydrol, 2003, 62-63: 637-652

7 张玉军. 遍有节理岩体的双重孔隙介质热-水-应力耦合模型及其有限元分析. 岩石力学与工程学报, 2009, 28: 947-955

8 梁冰, 刘否, 薛强, 等. 核素渗漏对地下水污染的数值仿真研究. 系统仿真学报, 2007, 19: 261-311

9 Sonnenthal E, Ito A, Spycher N, et al. Approaches to modeling coupled thermal, hydrological, and chemical processes in the drift scale heater test at Yucca Mountain. Int J Rock Mech Min Sci, 2005, 42: 698-719

10 武文华, 李锡㲘. 热-水力-力学-传质耦合过程模型及工程土障数值模拟. 岩土工程学报, 2003, 25: 188-192

11 刘泽佳, 李锡禀, 武文华. 多孔介质中化学-热-水力-力学耦合过程本构模型和数值模拟. 岩土工程学报, 2004, 26: 797-803

12 张玉军. 考虑溶质浓度影响的热-水-应力-迁移耦合模型及数值模拟. 岩土力学, 2008, 29: 212-218

13 Leiws R W, Schrefler B A. The Finite Element Method in the Static and Dynamic Deformation and Consolidation of Porous Media. Chichester, West Sussex: Wiley, 1998

14 Chijimatsu M, Kurikami H, Ito A, et al. Implication of THM coupling on the near-field of a nuclear waste repository in a homogeneous rock mass. DECOVALES III-Task3-Bench Mark Test 1(BMT1)-Subtask BMT1-B, 2002. 1—43

15 Nishigaki M. Density dependent transport analysis saturateds-unsaturated porous media-3 dimensional eulerian lagrangian method. Okayama University, 2001

16 Elsworth D, Bai M. Flow-deformation response of dual-porosity media. J Geotech Eng, 1992, 118: 107_124

17 Rutqvist J, Barr D, Datta R, et al. Coupled thermal-hydrological-mechanical analyses of the Yucca Mountain Drift Scale Test-Comparison of field measurements to predictions of four different numerical models. Int J Rock Mech Min Sci, 2005, 42: 680—697 